\title{
Technologists’ Forum: Safety in the Microscopy Laboratory
}

\author{
E. Ann Ellis ${ }^{1}$ and Beverly E. Maleeff ${ }^{2}$ \\ 1. Consultant in Biological Electron Microscopy, Thomasville, GA \\ 2. GlaxoSmithKline, King of Prussia, PA
}

Adherence to safety guidelines is a critical aspect of working in any laboratory, and particularly in microscopy laboratories where proper equipment use and maintenance, chemical handling, specimen handling and waste disposal are of the utmost importance. Microscopy labs often deal with hazardous materials such as osmium tetroxide and embedding media on an everyday basis. Safety education of the staff in these labs is essential for the health and safety for everyone involved with these labs.

Education is an essential part of all laboratory safety! In general, the most important aspect of laboratory safety is to read, understand and implement the regulations and standard operating procedures in place in that lab or institution. In addition, there may be local or federal laws that impact the conduct of laboratory research. It is the responsibility of each employee and his/her direct supervisor to ensure that all governing rules are provided and made clear before any work is undertaken.

Microscopy labs are often defined as either biological or materials science-oriented, dependent on the institution and the physical location of the equipment. While there are general laboratory rules that must be followed regardless of the types of specimens examined, each has rules and regulations specific to the discipline. For example, in a biological microscopy laboratory there must be biological hazard containers for sharps, broken glass, and discarded specimens; chemical fume hoods for the use of resin components; biological cabinets for handling unfixed animal tissues and fluids; spill kits; appropriate air handling systems; and appropriate signage on the outside of the laboratory door to notify anyone entering the laboratory about the proper personal protective equipment (PPE) needed to work safely. In a materials science laboratory, similar regulations must be in place, including containment systems specific to the type of specimens being prepared and examined.

Chemical fume hoods are essential components of all electron microscopy laboratories since osmium tetroxide and embedding resins are important but hazardous materials, used every day in the operation of the labs. Maintenance of fume hoods at appropriate operating levels is a continuous part of the housekeeping in that these facilities should be monitored daily for operation and not be allowed to become a cultured storage area for hazardous wastes. A Kimwipe ${ }^{\mathrm{TM}}$ check (tape a Kimwipe ${ }^{\mathrm{TM}}$ to the sash with the sash opened to the proper operating height) should be performed daily at the hood operating opening to have a visual clue to whether there is a proper directional airflow. In the event that there is no or reduced airflow, work should be stopped and the proper environmental health and safety staff contacted. Hood flow face velocities should be checked on a schedule determined by local regulations. Sash height at a face velocity of $100 \pm 20 \mathrm{ft}^{3} /$ minute should be marked on the outside of the hood along with the date that it was last checked [1]. This flow rate of $100 \mathrm{ft}^{3} / \mathrm{min}$ is adequate for toxic substances such as osmium, sodium cacodylate and lead and if the microscopist spends a major part of the work day using highly toxic chemicals, the flow rate should be increased to $150 \mathrm{ft}^{3} / \mathrm{min}$ [2].

Use of cryogens has increased as our specimen preparation procedures have evolved and this presents additional hazards from both freezing burns to potential asphyxiation. Liquid nitrogen evaporates to 
gaseous nitrogen which can displace room air and care must be taken to insure adequate ventilation in areas where liquid nitrogen is worked with and stored.

Radiation safety is equally important in a laboratory where radiation-generating instruments are used. In the United States, this is usually governed by state or local laws. At a minimum, this should consist of checking for radiation leakage at the time of installation, at yearly intervals thereafter, and at the time of any service including preventative maintenance. Any deviation from an expected reading must be reported immediately to your institutional radiation safety officer and corrected as soon as possible, and before the instrument is certified for use.

Waste disposal is a critical part of working in any laboratory, and no more important than in a microscopy lab. Biological and chemical waste must be addressed individually, and are typically handled using institutional or local regulations. Specific care must be taken with carcinogenic or toxic substances, including, but not limited to, resin components, sodium cacodylate, strong acids and bases, and the dust and shards generated from trimming resin blocks or materials samples.

Best laboratory practice includes the recording of all baseline equipment settings, equipment deviations, and maintenance and service records in appropriate log books or online databases so that any changes from baseline can be identified and corrected as required. In a lab that is subject to United States regulatory oversight, this is a legal requirement [3].

The most important safety rule is to use common sense! Plan your work, address the potential safety hazards associated with that work, and assemble everything needed to accomplish that work safely. If something does not seem right, then stop immediately and assess the situation. Contact your supervisor or safety officer before proceeding.

References:

[1] RS Stricoff and WB Walters. 1990. Laboratory health and safety handbook: a guide for the preparation of a chemical hygiene plan. John Wiley \& Sons, Inc., New York.

[2] VC Barber, 1994. in Electron Microscopy Safety Handbook,. VC Barber and JA Mascorro, eds. San Francisco Press, Inc., San Francisco. p. 3.

[3] Code of Federal Regulations, Title 21: Food and Drugs, Part 58: Good laboratory practice for nonclinical laboratory studies. US Food and Drug Administration.

[4] This paper does not necessarily reflect the approved practices of GlaxoSmithKline. 